\title{
Memory orientation and success: separable neurocognitive components underlying episodic recognition
}

\author{
Ian G. Dobbins ${ }^{\mathrm{a}, \mathrm{b}, *}$, Heather J. Rice ${ }^{\mathrm{b}}$, Anthony D. Wagner ${ }^{\mathrm{c}, \mathrm{a}}$, Daniel L. Schacter ${ }^{\mathrm{d}}$ \\ a Martinos Center for Biomedical Imaging, MGH/MIT/HMS, Charlestown, MA, USA \\ ${ }^{\mathrm{b}}$ Duke University, Durham, NC, USA \\ ${ }^{c}$ Massachusetts Institute of Technology, Cambridge, MA, USA \\ ${ }^{\mathrm{d}}$ Harvard University, Cambridge, MA, USA
}

\begin{abstract}
Episodic recognition can be based on recollection of contextual details, on a sense of recent encounter, or some combination of the two. According to several cognitive models, selectively attending to these distinct aspects of memory may require different retrieval orientations and result in different neural responses depending upon whether or not retrieval is successful. Using event-related fMRI, we examined retrieval orientation by having subjects discriminate between two test words in one of two manners. During source recollection, they selected the member of the pair previously associated with a particular encoding task. In contrast, recency judgment required selection of the most recently encountered item of the pair, regardless of how it had been encoded. Furthermore, successful and unsuccessful trials within each retrieval task were contrasted to determine whether retrieval success effects occurred in overlapping or dissimilar neural populations compared to those associated with each retrieval orientation. The results revealed distinct lateral prefrontal and parietal activations that distinguished attempted source recollection from judgments of relative recency; these orientation effects were largely independent of retrieval success. In contrast, medial temporal lobe structures (hippocampus and parahippocampal gyrus) were differentially more active during successful recollection of encoding context, showing similar reduced responses during failed source recollection and judgments of recency. These results indicate that different memory orientations recruit distinct prefrontal and parietal networks and that the recovery of episodic context is associated with the hippocampus and surrounding medial temporal cortices.
\end{abstract}

(C) 2002 Elsevier Science Ltd. All rights reserved.

Keywords: Event-related fMRI; Recollection; Familiarity; Source recognition; Recency; Retrieval

\section{Introduction}

Episodic recognition requires the judgment that an item has been previously encountered in one's personal past. Behavioral studies indicate that recognition judgments can depend on fundamentally different types of information. For example, under dual-process models of recognition test items can be used as retrieval cues to recover specific contextual details regarding the prior encounter, and this is termed recollection. In contrast, some items may yield a sensation of recent encounter, despite the fact that no contextual details are recollected [1-10]; this non-contextual form of recognition is usually termed item familiarity. Alternate characterizations also exist, which instead of focusing on process characteristics, draw a distinction be-

\footnotetext{
* Corresponding author. Present address: MGH-NMR Center, Department of Radiology, Bldg. 149, 13th Street, Charlestown, MA 02129, USA. Tel.: +1-617-724-9989; fax: +1-617-726-7422.

E-mail address: ian@nmr.mgh.harvard.edu (I.G. Dobbins).
}

tween recognizing the content versus the context of a prior event, or similarly between remembering what and when (e.g. $[11,12])$. Despite differing in several respects, these characterizations all raise the possibility that subjects may selectively orient attention towards different aspects of mnemonic information during testing (retrieval orientation), and that such orientation effects may be distinguishable from effects associated with successful recovery of the sought after mnemonic information (retrieval success).

In this article, we explore two central questions regarding episodic recognition. The first is whether the attempted retrieval of recollective source details results in a different retrieval orientation [13] than does attempted assessment of recency information that is orthogonal to source. More specifically, do subjects recruit different retrieval or monitoring processes depending on whether recollection of source details or recency information is most appropriate for a given memory task? The second question is whether we can further distinguish between successful and unsuccessful retrieval within each of these putative retrieval orientations. 
That is, within a particular orientation, are there neural responses that distinguish between successful and unsuccessful recovery of episodic information?

The primary framework motivating the current research was dual-process recognition theory, which suggests that recollection and familiarity processes are separable [5] and that in some retrieval situations, only one of the processes may be useful or necessary. If correct, then certain retrieval demands may steer subjects towards shifting attention to one or the other type of information during retrieval attempt. Extant neuroimaging data comparing source to item recognition implicate left prefrontal (PFC) and parietal cortical processes in tasks in which the retrieval of contextual specifics regarding source is essential and item familiarity is largely uninformative [14-16]. The designs of these studies, however, precluded a comparison of successful versus unsuccessful source performance making it unclear to what extent these neural computations are modulated by the success or failure of source recollection. A recent study partially addressing this issue revealed left anterior PFC activation during source retrieval that demanded recovery of perceptual detail relative to simple item recognition; this increase was observed irrespective of whether the test probe was studied or novel [17]. Because novel items should not yield recollection of contextual details, this finding suggests that the manner in which subjects engage test probes-and not their episodic histories-is reflected in these PFC responses. Again, however, no direct comparison between successful and unsuccessful source trials for studied items was possible.

In contrast to accounts focusing on the degree of specificity or reflective demands of the retrieval task, other theorists have argued that left PFC and parietal computations are specifically modulated by the successful recovery of episodic information. This perspective has been based on observations that activation is greater for hits relative to correct rejections [18-20]. Henson et al. [21] characterized activations in left ventral PFC regions as indirect indices of retrieval success, potentially subserving decision processes following successful retrieval. These findings, however, might appear at odds with the suggestion that PFC computations are engaged irrespective of the test probe's study history, and thus it is presently unclear as to whether the demand for a particular type of retrieval, or the outcome of the retrieval attempt, modulates left PFC.

There is also uncertainty regarding the characterization of right PFC responses during episodic retrieval. Tulving and colleagues suggested that right PFC responses are markers of episodic retrieval mode, which is characterized as a state in which current items are treated as retrieval cues with respect to a particular past episode [22]. In contrast, Henson and colleagues have suggested that right PFC is engaged in monitoring operations that are especially necessary when test items fail to yield useful recollective content and are close to the criterion for responding on the basis of familiarity (cf. [23]). Moreover, Wagner et al. have posited that these monitoring processes may be under strategic control, being recruited when task relevant [24].

We investigated differences in retrieval orientation and success across two episodic tasks by rendering the utility of task recollection and item recency orthogonal on a trial-by-trial basis, such that subjects were expected to adopt different retrieval orientations and to recover different forms of information for each task. If subjects can exert control over retrieval orientation, then cortical responses to retrieval cues requiring source discrimination (a recollection-based decision) will dissociate from those requiring the discrimination of relative recency, regardless of response accuracy. In addition, we sought to address this question using stimuli with identical encoding histories across the two tasks. In this manner, differential responses across the tasks could be attributed to different orientations or outcomes, but not to systematic differences in the composition of the test materials. In contrast, many previous comparisons of source and item recognition, or item and recency recognition, have used test items whose composition systematically differed across the different retrieval tasks. Therefore, observed differences may be attributable to the retrieval tasks, the different information afforded by the materials, or some combination of the two.

Based on prior research, the most likely locus for orientation differences across tasks is PFC. In particular, we predicted that when the test required the recollection of contextual specifics regarding the encoding task, heightened activation would be observed in left prefrontal and lateral parietal cortices. However, when this information was rendered non-diagnostic and subjects were required to discriminate the recency of the items, right prefrontal regions implicated in familiarity monitoring were expected to be more active $[14,21,25]$. This latter prediction receives some support from two prior studies of recency recognition employing positron emission tomography [11,12]. For example, Cabeza et al. [11] observed a greater PFC response during judgments of recency in comparison to item recognition and this increase was larger in the right than the left hemisphere. Greater PFC responses during recency were also observed by Zorrilla et al. [12]; however, the reference condition was a lexical matching task with all novel items, and therefore it is unclear whether the responses were unique to the mnemonic demands of the recency judgment, or instead simply reflected the greater working memory demands for the recency judgment. Furthermore, both studies used blocked designs and therefore item specific retrieval success was not examined.

An alternative prediction with regard to PFC is that the distinct retrieval tasks would not implicate separate prefrontal regions, but would instead show modulation of the same regions as a function of retrieval specificity. For example, as previously discussed, Ranganath et al. [17] found that increasing the perceptual specificity required during the retrieval task resulted in increases in anterior left PFC and attributed this to the need to monitor and evaluate specific 
memory characteristics at retrieval. Within the current study, requiring subjects to evaluate items in terms of relative recency might be viewed as a more specific memory judgment than discriminating between one of two possible sources. This is because each item is potentially associated with a unique recency value, whereas there are only two possible sources affiliated with the source task. If so, then one would predict that the recency task would yield greater activity in left PFC than the source task because of the finer grained demand of the retrieval task.

With respect to retrieval outcome, recognition tests that require the retrieval of contextual specifics may be particularly sensitive to hippocampal damage [26-32] and the hippocampal formation has been specifically postulated as a critical component in successful conscious episodic retrieval $[33,34]$. Thus, correct and incorrect source recognition should yield differential hippocampal activation. In contrast, from one perspective [28,35], retrieval success effects should not be observed within the hippocampus during recency recognition, as such judgments in the current paradigm are hypothesized to be largely non-recollective.

\section{Materials and methods}

\subsection{Overview of methods}

To address questions regarding the neural correlates of retrieval orientation and retrieval success, separate behavioral and event-related functional magnetic resonance imaging (fMRI) experiments were conducted. The general logic is shown in Fig. 1. Subjects studied a list of items while continually alternating between two semantic encoding tasks. Immediately following study, a two-alternative forced choice (2AFC) recognition test was given to separately assess source and recency recognition. For the recency judgment, subjects were asked to select the member of the pair seen most recently during the preceding study list. For the source judgment, they were asked to select the member with which they had performed a particular encoding task. Critically, the test list was constructed such that recollection of the specific encoding task performed for each word was non-diagnostic of which word had appeared most recently during prior study. Thus, recollection of the encoding source was irrelevant for determining recency. Likewise, knowledge of a word's relative recency was not correlated with the manner in which it had been encoded.

In the behavioral experiment, two manipulations were crossed with the source and recency tasks to assess whether performance on these tasks is doubly dissociable. These manipulations address the prediction that the source task requires cued recollection of item-specific contextual information, whereas, the recency task involves the assessment of recency-based differences that are not tied to the specific task previously performed with each of the items. The first manipulation was a systematic reduction in the study lag between the two words in each test pair, and the second involved the speed with which subjects were required to respond. Based on prior research, it was predicted that the lag manipulation would more severely impact recency

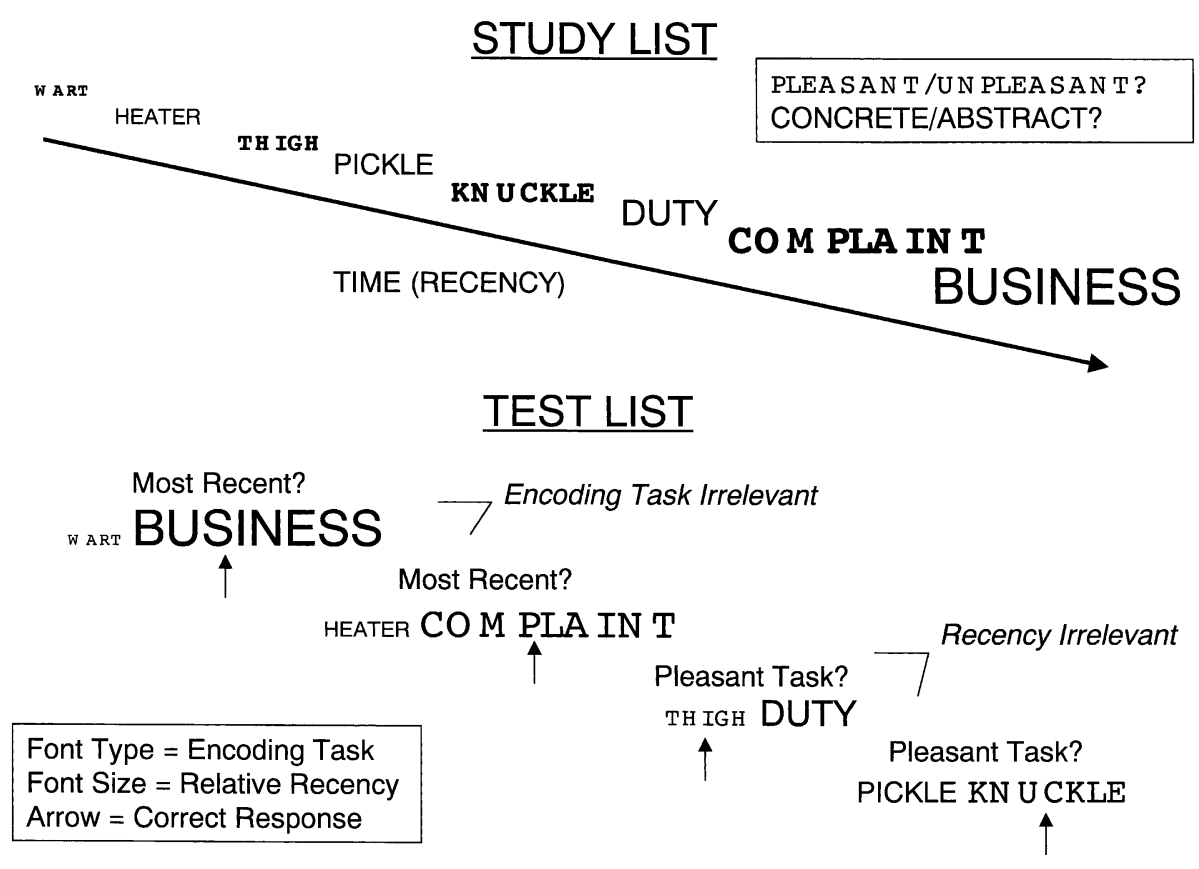

Fig. 1. The logic of the experimental design. Font size of the study items represents their degree of recency at testing. In contrast, the scrolled font represents those encoded using the pleasant/unpleasant task whereas the block font represents items encoded using the concrete/abstract task. The bottom half of the figure shows an example 2AFC test list and the independence of relative recency and encoding task. For illustrative purposes, small arrows indicate the correct response based on the retrieval cue. 
performance, whereas, the response deadline manipulation would more severely disrupt source performance $[1,7,36,37]$. Following the behavioral experiment, an event-related fMRI experiment was conducted to determine whether distinct neural networks are differentially recruited depending on (a) whether retrieval orientation is directed towards source recollection or recency judgment, and (b) whether or not retrieval was successful within each orientation.

\subsection{Subjects}

Fifteen native-English speaking volunteers (10 male; age range, 19-29 years) were paid US\$ 10 for participating in the behavioral study. Twelve different right-handed native-English speaking volunteers (eight male; age range, 19-26 years) were paid US\$ 50 for participating in the fMRI study. Data from one subject were excluded in the behavioral and imaging studies due to poor recognition. Informed consent was obtained in a manner approved by the Human Studies Committee at Massachusetts General Hospital and the Harvard University Committee on the use of Human Subjects in Research.

\subsection{Behavioral study materials}

A total of 640 nouns were drawn from the Oxford English Dictionary. From this list, four lists of 160 items were constructed for use in four separate study/test cycles. These lists did not differ in mean item length, syllables or word frequency [38]. Within each list, blocks of 40 words were serially rotated to counterbalance across conditions and subjects.

\subsection{Behavioral study procedure}

Subjects studied the list of items while alternating between two semantic orienting tasks (Fig. 1). One task required a pleasant/unpleasant decision for each item, whereas, the other required a concrete/abstract decision. Subjects were given $3 \mathrm{~s}$ to respond via keyboard to each visually presented item. Each encoding cue remained on the screen for the entire $3 \mathrm{~s}$. The study item appeared $250 \mathrm{~ms}$ after the onset of the cue and disappeared $250 \mathrm{~ms}$ before its removal.

Immediately following the study list, a two-alternative forced choice (2AFC) recognition test was administered to assess source and recency recognition. Source and recency recognition trials were randomly intermixed. Test pairs were constructed by systematically crossing the items from each half of the study list with the type of encoding that each received $\{$ lag (first or second half) $\mathrm{X}$ source (pleasantness or concreteness encoding)\}, yielding 80 test pairs in which one item was encoded for pleasantness, one for concreteness, and each differed in recency. Starting with the largest study lag separation (i.e. the first and 160th study items), the test pairs were alternately assigned to either a source or recency test condition in steps of two (i.e. 1-160, 2-159, 3-158 ... until 80-81) such that the type of retrieval cue (source or recency) was evenly distributed across the study lag separation. The order of presentation of pairs and side of correct response during testing was pseudo-randomized. The test cue to item pair correspondence was reversed for half of the subjects. For the relative recency judgment (i.e. most-recent?), subjects were asked to select the member of the pair seen most recently during the immediately preceding study list. For the source judgment, subjects were asked to select the member of the pair that they previously rated as pleasant or unpleasant (i.e. pleasant-task?).

Because recollection of the source context was irrelevant for determining which word in a pair occurred most recently and conversely the recency of each word in a pair was unrelated with the manner in which it had been encoded, it was predicted that the retrieval tasks could be dissociated. A behavioral study tested this prediction using two retrieval manipulations that were crossed with the source and recency tasks. The first manipulation was a systematic reduction in the study lag between the two words in test pairs. If the recency judgment is based on a differential signal that passively decays over time, then recency discrimination between items studied more closely in time should be more difficult than between items studied farther apart (i.e. items studied in closer proximity will have similar familiarity). This prediction is consistent with signal detection accounts of recognition [39]. In contrast, the lag manipulation was expected to have little impact on source recognition [40]. The second manipulation involved the response speed. Contextual recollection is held to be slower than familiarity assessment [1,7,36,37,41], and therefore speeding the recognition decision should have a more detrimental effect on source than on recency performance. In two of the four study/test cycles subjects were given $2 \mathrm{~s}$ to respond, in the remaining two they had $4 \mathrm{~s}$. Response speed alternated across study/test cycles. Stimulus presentation and responses were controlled using PsyScope [42].

\section{5. fMRI study materials and procedures}

The materials and tasks were identical to the behavioral pilot study with the following exceptions. A speeded recognition procedure was not used; instead, subjects were given $4 \mathrm{~s}$ to respond on all test trials. Responses were made with a magnet compatible button box, using the left index and middle fingers. Stimuli were back projected onto a screen at the rear of the magnet bore with subjects viewing the stimuli using a small rearward-facing mirror.

Each scanning subject received four study/test cycles and was only scanned during the tests. Prior to the first study list, and coincident with a T1-weighted structural scan, a brief practice run was conducted. The order of the test trial types (recency and source) within each test scan appeared random from the subjects perspective, but in actuality was determined using an optimal sequencing program designed 
to maximize the efficiency of recovery of the BOLD response [43]. Periods of visual fixation lasting between 2 and $10 \mathrm{~s}$, "jittered" in increments of $2 \mathrm{~s}$, were interspersed between trials as determined by the optimization. During fixation trials, positions were replaced by rows of crosses of approximately the same length as the stimuli.

\section{6. fMRI data acquisition}

Scanning was performed on a 3T Siemens Allegra system using a standard whole-head coil. Functional data were acquired using a gradient-echo echo-planar pulse sequence $(\mathrm{TR}=2 \mathrm{~s}, \mathrm{TE}=30 \mathrm{~ms}, 21$ axial slices parallel to the AC-PC plane, $3.125 \mathrm{~mm} \times 3.125 \mathrm{~mm} \times 5 \mathrm{~mm}, 1 \mathrm{~mm}$ inter-slice gap, 240 volumes per run). Prior to each scan, four volumes were discarded to allow for T1-equilibration effects. Each of the four scans lasted for $8 \mathrm{~min}$, and was preceded by an 8 min study list. High-resolution T1-weighted (MP-RAGE) anatomical images were collected for slice positioning. Head motion was restricted using a pillow and foam inserts that surrounded the head.

\section{7. fMRI data analysis}

Data were preprocessed using SPM99 (Wellcome Dept. of Cognitive Neurology, London). Images were corrected for slice timing, followed by rigid body motion correction. Functional data were spatially normalized to an MNI EPI template using 12-parameter affine and nonlinear cosine basis transformations. Images were resampled into $3-\mathrm{mm}$ cubic voxels and then spatially smoothed with an 8-mm FWHM isotropic Gaussian kernel; each session was re-scaled to a mean signal of 100 .

The data were analyzed treating subjects as a random effect. Volumes were treated as a temporally-correlated time series and modeled by convolving a synthetic hemodynamic response function and its first order time derivative with the appropriate onsets. The resulting functions were used as covariates in a general linear model, along with a basis set of cosine functions that were used to high-pass filter the data, and a covariate for session effects. The least squares parameter estimates of height of the best fitting synthetic HRF for each condition were used in pairwise contrasts and stored as a separate image for each subject. These images were then tested against the null hypothesis of no difference between conditions using one-sided $t$-tests. For the main comparison of source and recency tasks, a region was considered significant if it consisted of five or more contiguous voxels and exceeded an alpha threshold of $0.001(Z>3.09$, uncorrected). For accuracy (retrieval success or failure) comparisons, in which the number of observations in each condition for each subject was markedly reduced and for which the medial temporal regions of interest are known to provide a relatively modest signal to noise ratio compared to lateral neocortical structures, the threshold was lowered to an alpha of 0.005 ( $Z>2.58$, uncorrected) $[28,44-46]$.

\section{Results}

\subsection{Behavioral data}

The results demonstrated that the two retrieval manipulations (lag and response deadline) dissociated for accuracy between the source and recency tasks. A three-way within-subjects ANOVA, with factors of response deadline, recognition task, and study lag, demonstrated main effects of response deadline $(F(1,14)=20.41$, Mse $=0.014)$, Task $(F(1,14)=10.80$, Mse $=0.011)$, and item study lag $(F(1,14)=28.06$, Mse $=0.007)$. More importantly, there were task $\mathrm{x}$ response deadline $(F(1,14)=6.01$, Mse $=$ $0.006)$ and task X study lag $(F(1,14)=6.58$, Mse $=0.005)$ interactions. The task $\mathrm{X}$ deadline interaction occurred because speeding subjects' responses more severely disrupted source than recency performance (Fig. 2, top panel), whereas the task X study lag interaction occurred because decreasing the relative study lag between the members of a test pair more severely disrupted recency than source performance (Fig. 2, top panel). The three-way interaction was not significant $(F<1.0)$.

The fMRI study also yielded behavioral data (Fig. 2, bottom panels). The encoding and test procedures were similar to the behavioral study with one simplification; there was no response deadline manipulation during the imaging study. The manipulation of study lag yielded task X study lag interactions for accuracy $(F(3,30)=6.90$, Mse $=0.006)$ and median correct reaction time $(F(3,30)=51077$, Mse $=$ 12341). Simple effects contrasts for accuracy demonstrated that decreasing the study lag severely disrupted relative recency performance $(F(3,30)=9.54$, Mse $=0.006)$, but had no reliable effect on source accuracy $(F<1)$. Numerically, the interaction for reaction times suggested that decreasing the relative study lag slowed recency judgments while speeding source judgments. However, neither simple effect was statistically reliable. Collectively, these behavioral data provide strong evidence that the source and recency tasks rely on different mnemonic information and/or evaluation processes and therefore might dissociate neurally.

\section{2. fMRI data}

\subsubsection{Regions demonstrating similar activity for source and recency tasks}

Both the source and the recency tasks involve assigning mnemonic information to two items displayed on the screen, and maintaining that information until a selection is made. Given this similarity, it is likely that the two tasks will share verbal and/or spatial working memory components. To examine this possibility, we looked for regions showing joint signal increases, relative to fixation, for both tasks. Furthermore, regions which showed differential activity across the tasks, or which showed differential activity within each task as a function of retrieval outcome (successful or unsuccess- 

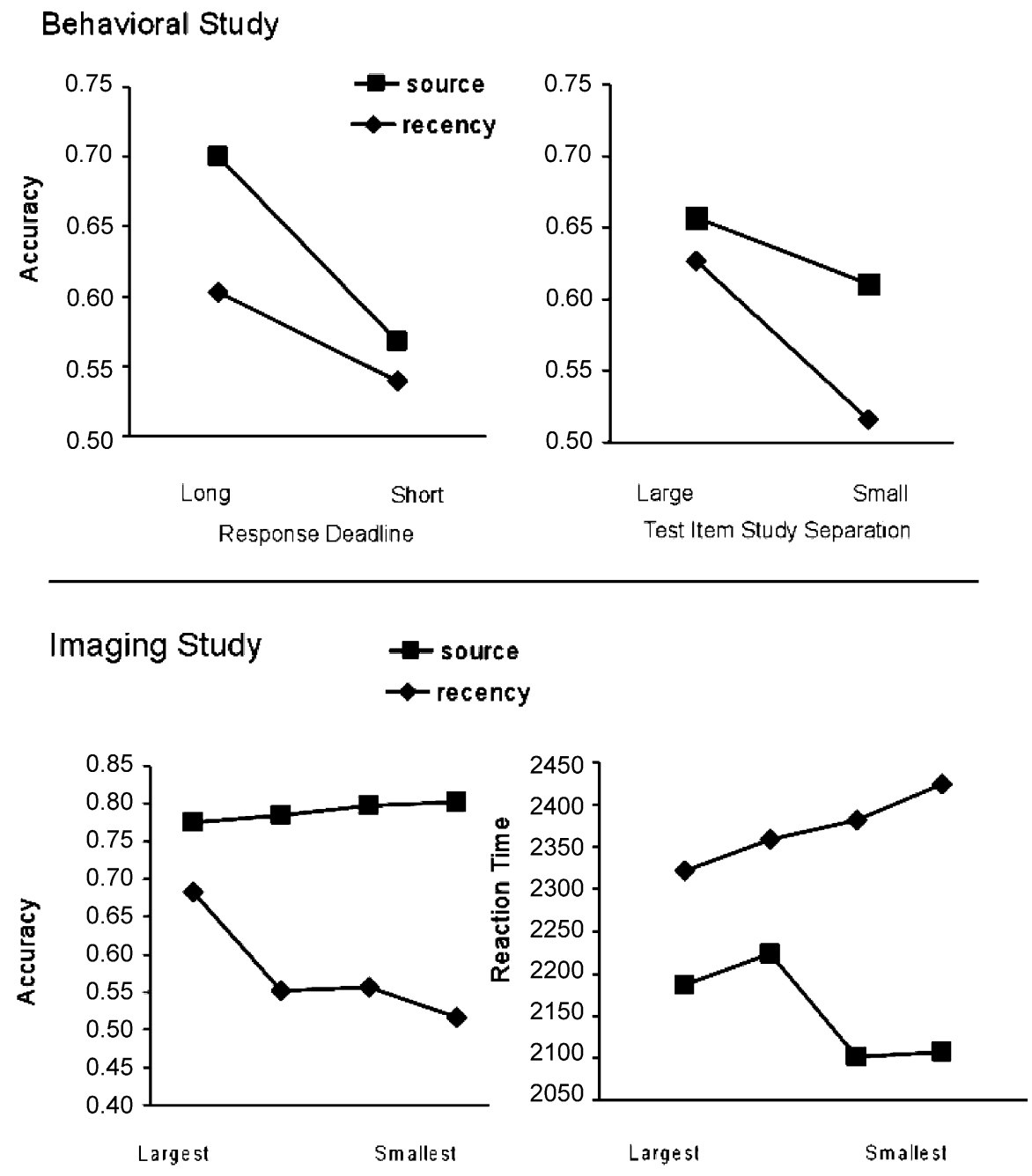

Test Item Study Separation

Fig. 2. Behavioral dissociations of accuracy for the source and relative recency tasks. Upper panels show the dissociations of source and recency accuracy across response deadline and study lag manipulations for the behavioral study. Lower left illustrates the dissociation of source and recency accuracy across the study lag manipulation for imaged subjects. Lower right illustrates the dissociation of source and recency median correct reaction time (ms) across the study lag manipulation for imaged subjects. The lag separation is plotted in quartiles (each 20 lag values) showing the progression from the largest (160 vs. 1 through 141 vs. 20$)$ to the smallest (100 vs. 61 through 81 vs. 80 ) lags.

ful) were excluded using masking (0.01 threshold, five voxel extent). This procedure ensured that the responses for the two tasks were highly similar in the identified regions.

The source and recency recognition tasks resulted in joint signal increases in prefrontal, parietal, and occipital regions relative to fixation. Most notably, there were bilateral increases in the dorsal aspect of the middle frontal gyri, lateral to the frontal eye fields (coordinates $-42,6,54$ and 33 , $6,48)$, and in the intraparietal sulci $(-24,-60,45$ and 36 , $-69,33)$. In addition, there was a joint increase in right posterior inferior PFC $(42,6,24)$. Because these regions failed to differentiate the retrieval tasks and were insensitive to retrieval outcome, they are likely involved in working memory or perhaps retrieval mode functions similarly recruited by both tasks and will not be considered further.

\subsubsection{Neurocognitive correlates of source recognition}

To explore the differential effects of distinct retrieval orientations, the two tasks were directly compared (Figs. 3 and 5; Table 1). Complementing the behavioral dissociation, comparison of the source task to the recency task yielded relative increases in numerous left-lateralized prefrontal, temporal-parietal, diencephalic and medial-temporal structures including left inferior frontal gyrus extending from the anterior and ventral portion of this region more dorsally and posteriorly into the caudal aspect of the middle frontal gyrus. Source recognition also yielded greater activation in left superior frontal gyrus, left lateral parietal cortex including supramarginal and angular gyri, posterior cingulate, bilateral caudate, left thalamus, and left hippocampus and parahippocampal gyrus (PHG). 

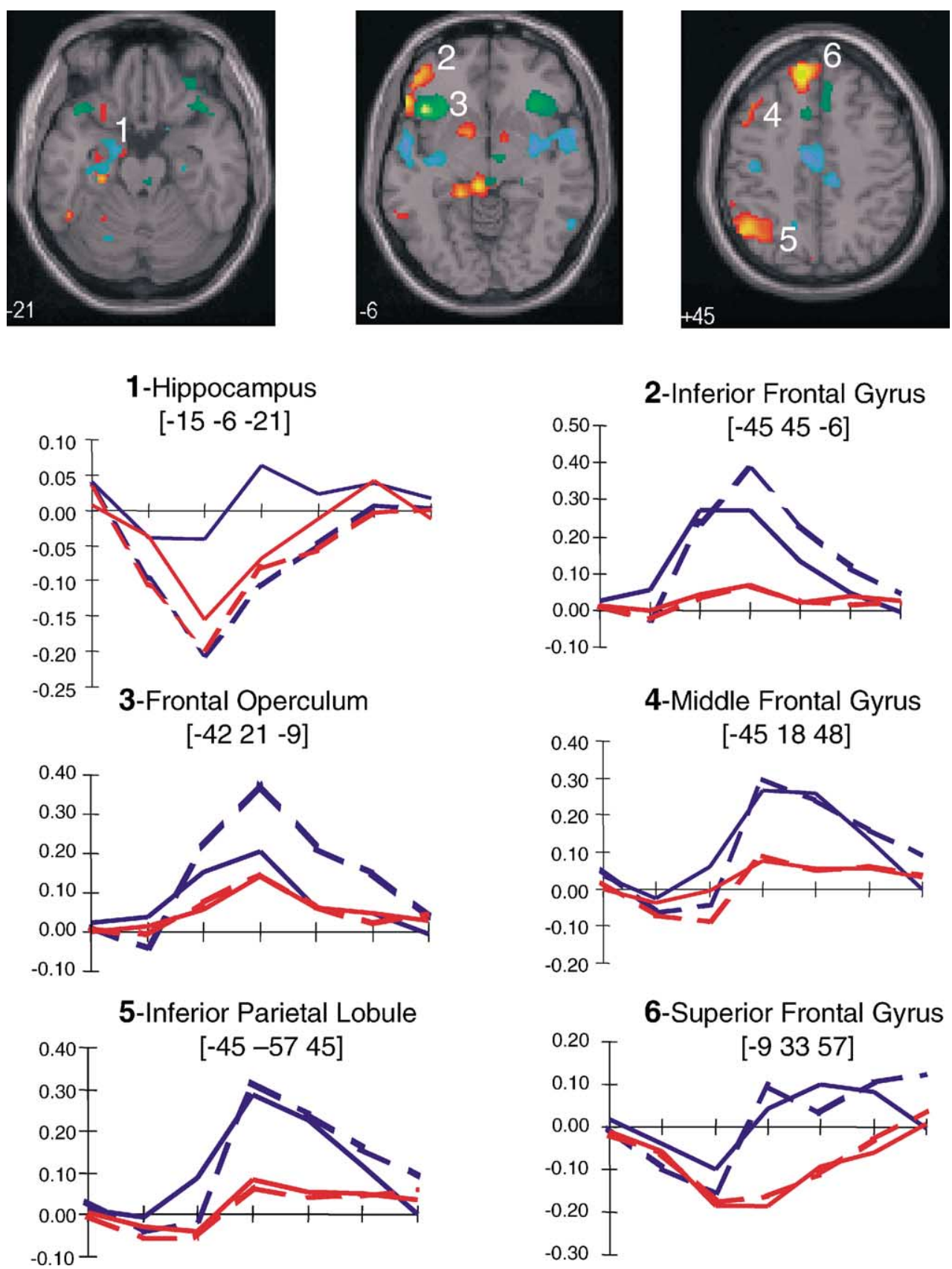

Fig. 3. Event related imaging results for the direct contrast of source vs. recency recognition rendered on a canonical brain. Regions in red/orange showed a greater response for source than recency and no differences as a function of response accuracy at 0.01 (retrieval orientation: $t$-values 4 or greater shown). Regions in cyan showed a greater response during correct vs. incorrect source (retrieval success: $t$-values 3 or greater shown). Regions in green showed an increased response during failed vs. correct source (retrieval failure: $t$-values 3 or greater shown). The line graphs show reconstructed hemodynamic responses from selected regions of interest (ROI). Ordinate is percent signal change, abscissa is post-stimulus onset time in increments of $2 \mathrm{~s}$. Blue lines represent source trials whereas red lines represent recency trials. Solid lines denote correct responding, dashed lines denote incorrect responding. Bracketed numbers are the maxima in MNI space for each ROI.

Importantly, within inferior frontal and posterior middle frontal gyri there was no evidence for a retrieval success effect even at a very liberal threshold ( 0.01 threshold, five voxels). In fact, these regions tended to respond similarly across successful and unsuccessful trials, or demonstrated the reverse pattern, that is, a greater response during source retrieval failure than success (Fig. 3). Of the 15 identified maxima extending along the left inferior frontal gyrus into posterior middle frontal gyrus, all showed a numerically higher peak response for unsuccessful compared to successful source responding. The only region within left PFC to demonstrate a greater response during successful than during unsuccessful source recognition was anterior superior frontal gyrus in an area close to that reported by Henson et al. [14] 
Table 1

Maxima of regions showing differential activation when comparing the source and recency tasks

\begin{tabular}{|c|c|c|c|c|c|c|c|}
\hline \multirow[t]{2}{*}{ Region } & \multicolumn{4}{|c|}{$\sim$ Talairach } & \multicolumn{3}{|c|}{ Brodmann } \\
\hline & $X$ & $Y$ & $Z$ & Hemisphere & NumVox & Area BA & Z-Score \\
\hline \multicolumn{8}{|l|}{ Source > recency } \\
\hline Superior frontal gyrus & -9 & 33 & 57 & Left & 42 & 6 & 5.26 \\
\hline Superior frontal gyrus & -9 & 45 & 45 & Left & 62 & 8 & 5.17 \\
\hline Superior frontal gyrus & -3 & 60 & 30 & Left & 40 & 9 & 4.18 \\
\hline Inferior frontal gyrus & -54 & 27 & -3 & Left & 47 & 47 & 5.23 \\
\hline Inferior frontal gyrus & -51 & 42 & 0 & Left & 36 & 46 & 4.63 \\
\hline Inferior frontal gyrus & -45 & 45 & -6 & Left & 62 & $47 / 11$ & 4.19 \\
\hline Inferior frontal gyrus & -45 & 21 & 33 & Left & 16 & 44 & 3.27 \\
\hline Middle frontal gyrus & -45 & 18 & 48 & Left & 40 & $8 / 9$ & 4.30 \\
\hline Posterior PHG & -27 & -27 & -21 & Left & 29 & 30 & 4.42 \\
\hline Posterior PHG/hippocampus & -18 & -36 & -9 & Left & 41 & 30 & 4.39 \\
\hline Hippocampus & -15 & -9 & -15 & Left & 18 & 35 & 3.67 \\
\hline Hippocampus & -33 & -18 & -12 & Left & 18 & 20 & 3.52 \\
\hline Hippocampus & -12 & -6 & -24 & Left & 15 & 28 & 3.32 \\
\hline Thalamus & -12 & -12 & 12 & Left & 61 & & 4.39 \\
\hline Caudate & 12 & 9 & 9 & Right & 44 & & 4.56 \\
\hline Inferior parietal lobule & -51 & -66 & 39 & Left & 32 & 39 & 4.45 \\
\hline Supramarginal & -60 & -48 & 30 & Left & 39 & 40 & 4.43 \\
\hline Inferior parietal lobule & -45 & -57 & 45 & Left & 74 & 40 & 4.40 \\
\hline Temporal pole & -54 & 3 & -33 & Left & 9 & & 4.09 \\
\hline Cerebellum & -48 & -51 & -21 & Left & 11 & & 3.94 \\
\hline Temporal pole & -48 & 15 & -36 & Left & 11 & 38 & 3.84 \\
\hline Temporal pole & -33 & 21 & -24 & Left & 17 & 38 & 3.76 \\
\hline Temporal pole & -24 & 15 & -24 & Left & 20 & 38 & 3.67 \\
\hline Posterior middle temporal & -57 & -48 & -9 & Left & 12 & 37 & 3.51 \\
\hline Precuneus & -9 & -75 & 33 & Left & 49 & $7 b / 19$ & 4.26 \\
\hline \multicolumn{8}{|l|}{ Recency > source } \\
\hline Transverse temporal gyrus & -60 & -15 & 12 & Left & 24 & 42 & 5.07 \\
\hline Insula & 45 & -12 & 6 & Right & 7 & 13 & 4.17 \\
\hline Insula & -39 & -21 & 3 & Left & 26 & 13 & 4.00 \\
\hline Superior temporal gyrus & -45 & -15 & -3 & Left & 18 & 22 & 3.54 \\
\hline Superior temporal gyrus & -48 & -24 & 3 & Left & 22 & 22 & 3.48 \\
\hline Insula & -36 & -12 & 21 & Left & 7 & 13 & 3.92 \\
\hline Cingulate & 9 & -36 & 36 & Right & 13 & 31 & 3.84 \\
\hline Superior frontal gyrus & 21 & 57 & 0 & Right & 6 & 10 & 3.84 \\
\hline Middle frontal gyrus & 27 & 39 & 36 & Right & 18 & 9 & 3.77 \\
\hline Superior frontal gyrus & 18 & 42 & 33 & Right & 7 & 9 & 3.30 \\
\hline Middle frontal gyrus & 33 & 33 & 45 & Right & 3 & 8 & 3.14 \\
\hline Postcentral gyrus & 39 & -27 & 39 & Right & 8 & 2 & 3.74 \\
\hline Inferior parietal lobule & 33 & -33 & 39 & Right & 5 & 40 & 3.39 \\
\hline Postcentral gyrus & 54 & -33 & 51 & Right & 18 & 40 & 3.74 \\
\hline Superior temporal gyrus & -51 & -30 & 15 & Left & 7 & 29 & 3.50 \\
\hline Superior temporal gyrus & -51 & -6 & 0 & Left & 10 & 22 & 3.49 \\
\hline Insula & 36 & -15 & 15 & Right & 5 & 13 & 3.44 \\
\hline Middle frontal gyrus & 27 & 21 & 57 & Right & 8 & 6 & 3.43 \\
\hline
\end{tabular}

Analyses were thresholded at 0.001, uncorrected.; PHG: parahippocampal gyrus. A list of all coordinates is available from authors by request.

to show a greater response for correct "remember" versus "know" judgments. Finally, among other regions, bilateral anterior insular/opercular cortices showed the reverse pattern, an elevated response during failed compared to successful source recognition (Table 2). Collectively, these results demonstrate the distinction between retrieval orientation and retrieval success effects. Regions engaged during a particular type of orientation differentiated between different retrieval tasks regardless of outcome. Furthermore, within a particular retrieval task these regions demonstrated similar or elevated responses during failed compared to successful retrieval, since the orientation must be maintained until recovery is successful or the trial is terminated. Left anterior inferior frontal gyrus, posterior middle frontal gyrus, superior frontal gyrus, and lateral parietal cortex demonstrated these characteristics and are therefore likely to be involved in operations necessary for initiating source retrieval attempts or monitoring the products of such attempts (Fig. 3 regions 2, 4-6).

Bilateral opercular regions showed elevated responses during failed source trials but did not distinguish between 
Table 2

Maxima of regions showing differential activation across source retrieval accuracy (analyses were thresholded at 0.005 , uncorrected)

\begin{tabular}{|c|c|c|c|c|c|c|c|}
\hline \multirow[t]{2}{*}{ Region } & \multicolumn{4}{|c|}{$\sim$ Talairach } & \multicolumn{3}{|c|}{ Brodmann } \\
\hline & $X$ & $Y$ & $Z$ & Hemisphere & Voxels & Area BA & Z-Score \\
\hline \multicolumn{8}{|l|}{ Source hit $>$ miss } \\
\hline Postcentral gyrus & 63 & -21 & 27 & Right & 54 & 2 & 4.69 \\
\hline Transverse temporal gyrus & 63 & -18 & 12 & Right & 53 & 42 & 3.85 \\
\hline Middle temporal gyrus & -57 & -30 & 3 & Left & 45 & 22 & 4.28 \\
\hline Superior temporal gyrus & -54 & -6 & -3 & Left & 59 & 22 & 4.23 \\
\hline Retrospenial & -6 & -36 & 6 & Left & 36 & 30 & 4.17 \\
\hline Insula & -36 & -15 & -3 & Left & 41 & 13 & 4.06 \\
\hline Insula & -36 & 3 & 0 & Left & 14 & 13 & 3.12 \\
\hline Superior frontal gyrus & -21 & 45 & 39 & Left & 19 & 8 & 4.01 \\
\hline Superior frontal gyrus & -15 & 54 & 36 & Left & 12 & 9 & 3.42 \\
\hline Cuneus & -12 & -81 & 30 & Left & 41 & 18 & 4.00 \\
\hline Cuneus & 3 & -87 & 21 & Right & 21 & 18 & 3.15 \\
\hline Paracentral lobule & -6 & -12 & 45 & Left & 39 & 31 & 3.76 \\
\hline Paracentral lobule & 12 & -27 & 45 & Right & 28 & 6 & 3.67 \\
\hline Postcentral gyrus & -27 & -30 & 54 & Left & 22 & 3 & 3.66 \\
\hline Hippocampus/entorhinal & 27 & -12 & -27 & Right & 23 & 36 & 3.50 \\
\hline Perirhinal/entorhinal & -24 & -18 & -30 & Left & 20 & 36 & 3.18 \\
\hline Hippocampus/amygdala & -15 & -6 & -21 & Left & 20 & 28 & 3.16 \\
\hline Hippocampus/entorhinal & -24 & -18 & -15 & Left & 20 & 20 & 2.97 \\
\hline Posterior PHG & -33 & -30 & -15 & Left & 9 & 20 & 3.02 \\
\hline Inferior temporal gyrus & 54 & -57 & -3 & Right & 19 & 37 & 3.46 \\
\hline Posterior middle temporal gyrus & 45 & -69 & 9 & Right & 30 & 37 & 3.45 \\
\hline Posterior middle temporal gyrus & -54 & -66 & 6 & Left & 33 & 37 & 3.56 \\
\hline Superior parietal lobule & -27 & -45 & 60 & Left & 25 & 7 & 3.44 \\
\hline Superior parietal lobule & -36 & -42 & 63 & Left & 26 & 5 & 3.10 \\
\hline \multicolumn{8}{|l|}{ Source miss $>$ hit } \\
\hline Inferior frontal gyrus & -42 & 21 & -9 & Left & 68 & 47 & 5.06 \\
\hline Inferior frontal gyrus & -30 & 24 & -6 & Left & 56 & 47 & 3.90 \\
\hline Middle frontal gyrus & -39 & 33 & -12 & Left & 44 & $47 / 11$ & 3.44 \\
\hline Inferior frontal gyrus & 39 & 24 & -15 & Right & 72 & 47 & 4.23 \\
\hline Inferior frontal gyrus & 33 & 24 & -9 & Right & 70 & 47 & 4.09 \\
\hline Medial frontal gyrus & 9 & 30 & 33 & Right & 39 & 9 & 3.82 \\
\hline Superior frontal gyrus & 3 & 48 & 30 & Right & 14 & 9 & 3.30 \\
\hline Medial frontal gyrus & 6 & 24 & 45 & Right & 20 & 8 & 3.09 \\
\hline Caudate & 9 & 9 & 9 & Right & 18 & & 3.43 \\
\hline
\end{tabular}

PHG = parahippocampal gyrus. A list of all coordinates is available from authors by request.

source and recency tasks during successful responding (Fig. 3, region 3). Thus, activity in this region is not unique to the source task, but elevated activity occurred for source retrieval failure. Similar prominent bilateral opercular activations were seen by Rugg et al. [47] in a comparison of source and simple item recognition [47], and by Buckner et al. [48] in a comparison of less and more successful recognition [48]. However, because these earlier studies used blocked designs, it is unclear whether those activations were also largely the outcome of source retrieval failure. In addition, this region may respond more generally during the failure to retrieve declarative knowledge, even when this information is non-episodic (e.g. semantic knowledge). For example, Maril et al. [49] observed bilateral opercular responses during the tip-of-the-tongue (TOT) phenomenon, a retrieval state in which subjects fail to retrieve target semantic knowledge such as a well-known name, but feel that they are on the verge of successful retrieval [49].
Finally, medial temporal lobe structures (greater on the left)—including hippocampus, posterior PHG, and rhinal cortex demonstrated greater responses during successful as opposed to unsuccessful source recognition (Fig. 3, region 1; Table 2). With the exception of the perirhinal/entorhinal focus, these regions demonstrated (a) an overall greater response during source than recency trials and (b) activation during recency trials (regardless of outcome) that tracked unsuccessful source responses. By contrast, overall activation in the perirhinal/entorhinal focus did not differentiate between source and recency judgments, although this region also demonstrated a source success effect. Thus, activation in the hippocampus and PHG was clearly selective for source retrieval success, with perirhinal cortex demonstrating a more complex pattern. Eldridge et al. [28], using the "remember/know" paradigm, observed a conceptually similar pattern [28]. In that study, only correct endorsements accompanied by subjective reports of remembering resulted 
in hippocampal activation. Correct "know", correct rejections, and miss trials, all of which are presumably made in the absence of recollection, showed similar and reduced hippocampal responses. In the present study, success-related differences were additionally observed in superior temporal, insular, middle cingulate, left cuneus, and left superior frontal areas (Table 2).

These results not only support a distinction between orientation and retrieval outcome, but also suggest functional heterogeneity within left PFC and between left PFC and medial temporal regions. This hypothesis was confirmed by examining the peak response ( $6 \mathrm{~s}$ post-stimulus onset) for three of the regions discussed above (posterior middle frontal gyrus, left frontal operculum, and left hippocampus) with ANOVA (Fig. 4A). A three-way ANOVA, with factors of region, task (source or recency judgment) and outcome (hit or miss), revealed a significant three-way interaction $(F(2,20)=21.48$, Mse $=0.002, P<0.0001)$. This interaction occurred because response outcome did not interact with the regions (in either direction) during recency judgments $(F<1)$ whereas, there was a strong interaction between outcome and regional response during the source task $(F(2,20)=36.72$, Mse $=0.003, P<0.0001)$. The hippocampus demonstrated an elevated response during source success $(P<0.005)$, the operculum showed a decreased response $(P<0.001)$, and the posterior middle frontal gyrus was unaffected $(P=0.34)$.

\subsubsection{Neurocognitive correlates of recency recognition}

The comparison of recency to source revealed activations that were primarily lateralized to the right hemisphere (Table 1). In particular, there was greater activation along the right superior frontal, right anterior prefrontal/frontopolar,
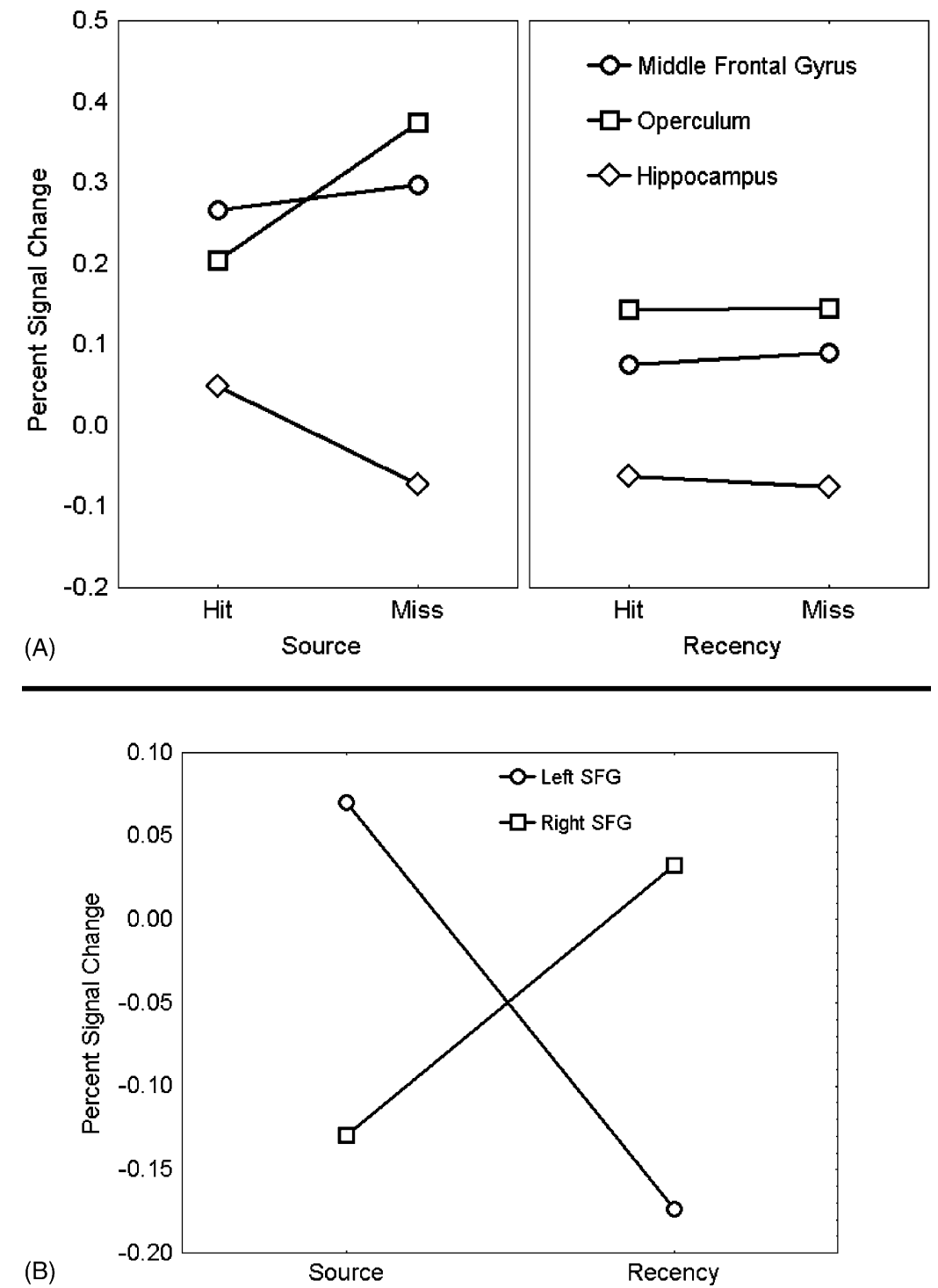

Fig. 4. (A) Regional interaction between left posterior middle frontal gyrus $(-45,18,48)$, frontal operculum $(-42,21,-9)$ and hippocampal formation $(-15,-6,-21)$ as a function of retrieval task (source vs. recency) and outcome (success or failure). (B) Hemispheric interaction between left $(-9,33$, 57 ) and right $(27,21,57)$ superior frontal gyrus as a function of type of retrieval task. Data reflect the peak response at $6 \mathrm{~s}$ post-stimulus onset. 
right supramarginal, left superior temporal, and right posterior cingulate cortices. Again, as with many of the source regions, these activations were largely unaffected by retrieval outcome even at a liberal threshold (0.01 threshold, 5 voxels; Fig. 5). Because overall performance was quite low during the recency task, the retrieval outcome results should be treated as conservative; a potentially larger network of regions might be implicated with additional power. Interestingly, the comparison of recency misses to recency hits yielded increased activation in right dorsolateral PFC and anterior cingulate (Table 3). Henson et al. [25] suggested that right dorsolateral PFC is involved in monitoring processes that operate when familiarity values are close to the decision criterion. They reported activity in this region when contrasting low versus high confidence hits. The current data partially support this notion by demonstrating an increased response during failed recency trials in which the familiarity of the items was likely more similar than during successful recency trials.
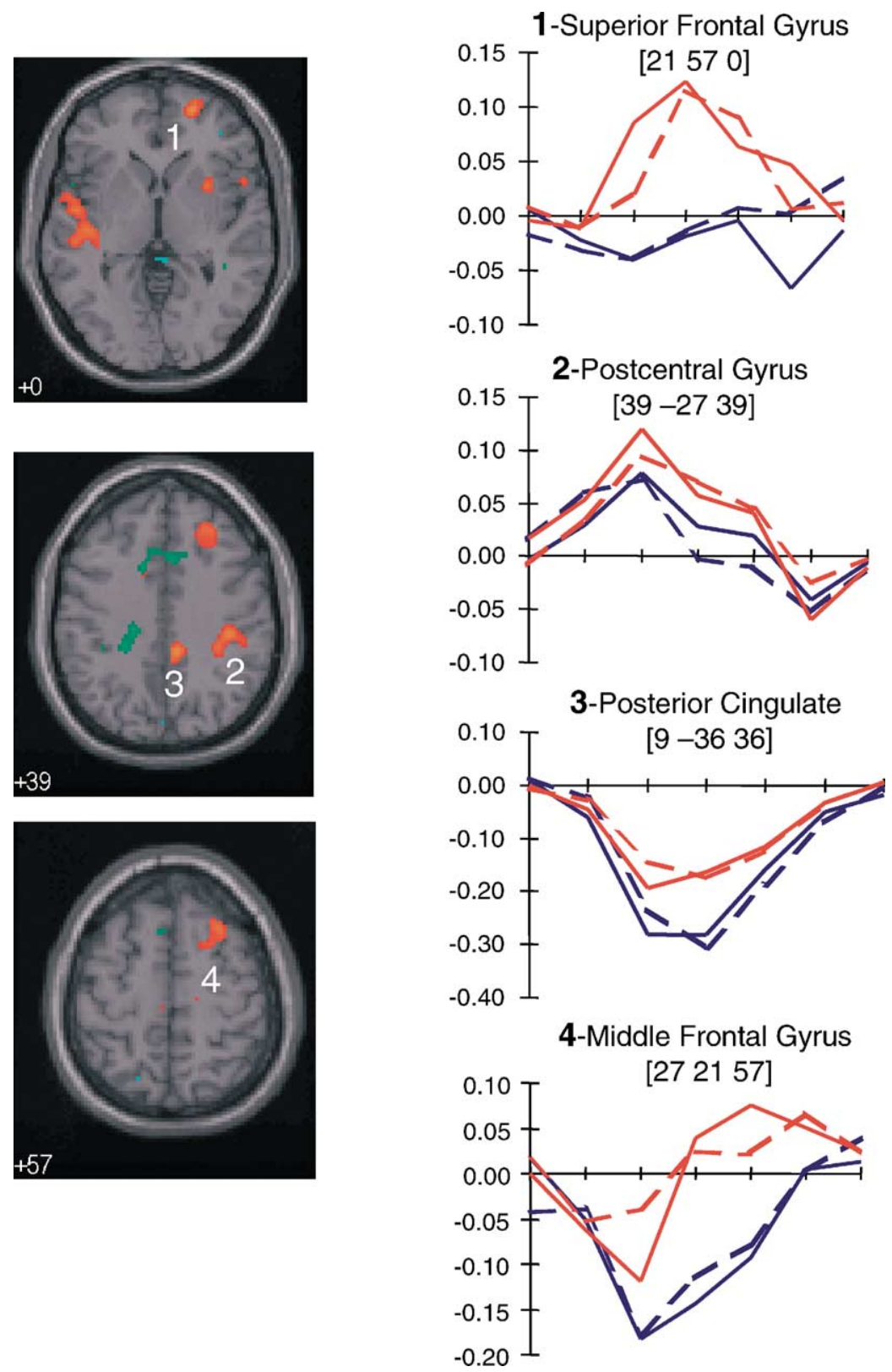

Fig. 5. Event related imaging results for the direct contrast of recency vs. source recognition. Regions in red/orange showed a greater response for recency than source and no differences as a function of response accuracy at 0.01 (retrieval orientation: $t$-values 3 or greater shown). Regions in cyan showed a greater response during correct vs. incorrect recency (retrieval success: $t$-values 3 or greater shown). Regions in green showed an increased response during failed vs. correct recency (retrieval failure: $t$-values 3 or greater shown). The line graphs show reconstructed hemodynamic responses from selected ROIs. Ordinate is percent signal change, abscissa is post-stimulus onset time in increments of $2 \mathrm{~s}$. Blue lines represent source trials whereas red lines represent recency trials. Solid lines denote correct responding, dashed lines denote incorrect responding. 
Table 3

Maxima of regions showing differential activation across recency retrieval accuracy (analyses were thresholded at 0.005 , uncorrected)

\begin{tabular}{|c|c|c|c|c|c|c|c|}
\hline \multirow[t]{2}{*}{ Region } & \multicolumn{4}{|c|}{ 〜 Talairach } & \multicolumn{3}{|c|}{ Brodmann } \\
\hline & $X$ & $Y$ & $Z$ & Hemisphere & Voxels & Area BA & Z-Score \\
\hline \multicolumn{8}{|l|}{ Recency hit > miss } \\
\hline Middle frontal gyrus & 48 & 15 & 21 & Right & 5 & 44 & 3.71 \\
\hline Superior frontal gyrus & 33 & 57 & -6 & Right & 6 & $10 / 11$ & 3.47 \\
\hline Precentral gyrus & -18 & -21 & 75 & Left & 6 & 4 & 3.30 \\
\hline \multicolumn{8}{|l|}{ Recency miss > hit } \\
\hline Medial frontal gyrus & -9 & 30 & 30 & Left & 26 & 9 & 4.48 \\
\hline Cingulate & 6 & 18 & 39 & Right & 17 & 32 & 3.15 \\
\hline Superior frontal gyrus & 39 & 36 & 30 & Right & 28 & 9 & 3.76 \\
\hline Polar middle temporal gyrus & -57 & 3 & -30 & Left & 10 & 21 & 3.37 \\
\hline Medial frontal gyrus & 6 & 63 & 12 & Right & 7 & 10 & 3.03 \\
\hline Superior frontal gyrus & -6 & 24 & 51 & Left & 15 & 8 & 3.02 \\
\hline
\end{tabular}

\subsubsection{Considering "task difficulty" and "effort"}

Although the lag manipulation was designed to dissociate source and recency performance by differentially driving down recency accuracy, inspection of Fig. 2 shows that subjects had considerable difficulty discriminating the test pairs for relative recency, except at the largest study lag separations. This observation raises concerns that the retrieval orientation differences were attributable to differences in relative task difficulty, or that the right PFC increases for recency reflect activations associated with non-mnemonic guessing strategies. To examine this, we contrasted source and recency trials during the largest study lag separation, during which source and recency performance was more comparable ( 0.78 versus 0.68 , respectively). The PFC differences previously observed across all lags were also preserved when selectively considering this largest lag. Critically, in comparison to source recognition, recency recognition again yielded greater activation in right anterior PFC (coordinates of 25, 54, 0) and right middle frontal gyrus (27, 39, 39). Furthermore, the same right frontopolar and middle frontal regions were activated in the comparison of recency misses to source misses ( 0.005 , five voxels).

These data provide two reasons to doubt an effort or difficulty based account of the current PFC differences. First, for unsuccessful responding on both tasks it can be argued that the tasks were similarly difficult, yet the PFC differences were preserved. Second, the pattern was preserved even when performance levels were more comparable. This observation suggests that the differences arose not because the subjects were on average more successful during one task than the other, but rather because they oriented towards the test materials in a fundamentally different manner due to the differential diagnostic value of cued recollection and recency information. A final reason to doubt a relative difficulty account can be seen in Fig. 4B. Referring back to Fig. 3 region 6 and Fig. 5 region 4, it can be seen that these two areas fell in roughly homogolous areas of left and right dorsal superior frontal gyrus. Nonetheless, they showed what appears to be mirror reversed hemodynamic response patterns.
Whereas the region in left superior frontal gyrus demonstrated a relative increase for the source task, the region in right superior frontal gyrus demonstrated a relative increase for the recency task; neither region was affected by task outcome (Fig. 4B). This was confirmed with an ANOVA employing factors of hemisphere (left or right), Task (source and recency), and Outcome (hit or miss). The only significant outcome was a strong hemisphere $\mathrm{X}$ task cross-over interaction, $(F(1,10)=66.02$, Mse $=0.01, P<0.0001)$. This result argues against a relative difficulty account in explaining the hemispheric differences across the tasks. These data also strengthen earlier findings of increased left PFC activity for comparisons of source and item recognition $[15,47]$. One concern regarding these earlier findings has been that source recognition is more "difficult" than item recognition. The current data have identified a similar source related network even when the reference memory task was behaviorally more difficult; this again suggests that these PFC responses are not markers of relative task difficulty (see also [50]).

\section{Discussion}

The present experiments demonstrated different retrieval orientations on a trial-by-trial basis depending on the utility of source versus recency information. The different patterns of neural activation across the recognition tasks were evident for test stimuli with identical study histories and, for most PFC regions, did not increase as a function of successful responding. Thus, the differential responses depended on the manner in which subjects oriented towards the test materials, and not on the history of the stimuli themselves or on the successful recovery of mnemonic information. The data further isolated these retrieval orientation effects from those associated with retrieval success. The most notable retrieval success effects were in bilateral hippocampal and PHG regions (greater on the left), which demonstrated a greater hemodynamic response during successful as opposed to unsuccessful source recognition. It should be re-emphasized 
that a demonstration of different retrieval orientations requires at least two simultaneous criteria to be met: (1) the region associated with a particular orientation should show a differential response across the tasks regardless of outcomes (success or failure), and (2) within the region, the response during failure should be equivalent or greater than during success for the affiliated task. The present data clearly demonstrate that the processes recruited during different retrieval orientations are largely separable from those marking the successful recovery of item-specific episodic detail. In addition, the data also demonstrated functional heterogeneity across regions of left PFC and between left PFC and the left hippocampal region by identifying differences in sensitivity to source retrieval outcome across these regions.

Our functional imaging results build on extant data investigating correlates of recollection and familiarity using the remember/know $(\mathrm{R} / \mathrm{K})$ procedure. Strikingly, almost all regions associated here with increases during source as opposed to recency recognition were identified by Henson et al. [14] in a comparison of "remembered" items to correctly rejected new items [21]. These include left superior, inferior, and middle frontal gyri, and left parietal, posterior cingulate and precuneus cortices. Similarly, those regions identified by Henson et al. were virtually exclusively left lateralized. Henson et al. characterized the left ventral and dorsal PFC regions as indices of general retrieval success and as potentially subserving post-retrieval decision processes. Likewise, Konishi et al. [19] observed greater activation in left inferior PFC and lateral parietal regions during recognition hits relative to correct rejections and theorized that these structures are involved in success-related processes following episodic retrieval [19]. However, in the present study, activation in these regions did not increase during successful compared to unsuccessful source recognition. In fact, these regions tended to show a numerically reduced peak response during successful responding and in the case of posterior inferior frontal gyrus ( $\sim$ BA 44$)$ this difference was significant (Table 2). This finding provides strong evidence that these regions are not indicators (direct or indirect) of episodic retrieval success (Fig. 3). Instead, these regions appear to be influenced by whether or not subjects are attempting to retrieve recollective content in response to the recognition task [51], an orientation effect that is governed by situational demands.

The suggestion that left prefrontal and parietal regions reflect a retrieval orientation specific to recollective attempt also fits well with other recent findings [14,17]. For example, in a comparison of an "exclusion" recognition condition, where only items from a particular source were to be endorsed, to an "inclusion" recognition condition, where all studied items were to be endorsed regardless of source, Henson et al. [14] found-increases in bilateral PFC and left parietal cortex consistent with the greater demand to monitor the materials for contextual recollection during the exclusion task [14]. Although the focus of that article was on interpreting the right $\mathrm{PFC}$ activation in light of its potential contribution to retrieval monitoring, the finding of left PFC and parietal regions proximal to those reported herein, is also consistent with an increased need to maintain a recollective retrieval orientation "exclusion" versus "inclusion" tasks. Similarly, Ranganath et al. [17] observed increased left PFC responses when contrasting a recognition paradigm requiring close monitoring of source specific perceptual information to a standard item recognition paradigm [17]. This increase was present for old items, and for new items that typically cannot yield episodic information, supporting our contention that left anterior inferior PFC responses mark a recollective retrieval orientation rather than recollective success.

The apparent divergence between our results and prior studies naturally leads to the question of why previous studies contrasting correct responses to old items (hits) and to new items (correct rejections) often found differences in the engagement of several of these regions [18-20]. For example, Konishi et al. [19] contrasted hits and correct rejections and found increased activity in left anterior prefrontal, bilateral frontal opercula, thalamus, left premotor, lateral parietal and precuneus regions and suggested these areas may represent a network correlated with episodic retrieval success. This idea follows from the logic that episodic information cannot be recovered for new items and, hence, the comparison of hits and correct rejections should isolate regions related to episodic recovery. The present study suggests, however, that many of these regions are not markers of episodic retrieval success, at least with respect to the kind of episodic information necessary for source discrimination. Indeed, the frontal opercula and posterior inferior frontal gyrus demonstrated the opposite of a success effect-they showed increased activity during failed source attempts. In addition, the left anterior inferior frontal gyrus, left superior frontal gyrus, left lateral parietal, and left posterior middle frontal gyrus demonstrated remarkably similar time courses across successful and unsuccessful source performance, and all the regions, with the exception of the opercula, were selectively engaged during the source as compared to the recency task (Table 1).

One possible explanation for the discrepancy across the present and prior studies involves the fact that whereas the current design matched the study history of items and manipulated retrieval tasks (source and recency), prior studies contrasted items with different histories (studied and novel) across the same task (old/new recognition). According to numerous dual-process models of recognition [4-6,10,52], old and new items differ not only in terms of familiarity, but also in terms of their usefulness in cuing contextual recollections. Because recollection is typically characterized as a strategic act, subjects may not attempt recollection for items perceived as relatively novel or unfamiliar because such attempts would likely be unproductive [1]. Indeed, the correct rejection of novel items is often conducted very rapidly and confidently, which is consistent with the idea that low familiarity items may often fail to invoke recollection attempts $[7,39]$. 
In view of these ideas, the comparison of hits and correct rejections may likely contain a systematic difference in retrieval orientation as well as a difference in retrieval success. It is therefore uncertain whether prior differences arising from comparisons between hits and correct rejections are (a) indicative of retrieval success, (b) reflect the greater tendency of subjects to adopt a recollective retrieval orientation for familiar as opposed to unfamiliar test items, or (c) represent some combination of the two. Our data provide strong evidence that modulations in several left PFC and left parietal cortices reflect recollective orientation independent of retrieval success. One area previously identified by Konishi et al. [19] in which we also found evidence for an episodic retrieval success effect was cuneus/precuneus, which in the present study demonstrated an increased response during successful compared to unsuccessful source responding. The other main structures demonstrating an episodic retrieval success effect, to which we now turn, were the hippocampus and PHG.

A marker of recollective retrieval success using the $\mathrm{R} / \mathrm{K}$ paradigm was obtained by Eldridge et al. [28], who noted increased bilateral hippocampal responses when subjects accurately reported "remembering" in comparison to correct "know", correct rejection, and miss responses; the latter three conditions resembled one another in response amplitude [28]. Eldridge et al. inferred from these results that the hippocampus is activated only when retrieval is accompanied by conscious recollection of the encoding context (see also [31]). Neuroanatomically, their foci resemble those that we found to differentiate between accurate versus inaccurate source responses. Our data further support the recollection hypothesis because inaccurate source responses as well as accurate and inaccurate recency responses showed similarly reduced levels of activation in left MTL regions, compared with accurate source responding (see Fig. 3). These findings indicate that successful recovery of prior contextual information critically depends on the hippocampal region and is possibly preceded by the adoption of a recollective retrieval orientation associated with left PFC and lateral parietal regions. This interpretation also receives support from a recent neuropsychological study contrasting patients with left and right MTL damage using the "remember/know" procedure [53]. Moscovitch and McAndrews [53] found that damage to the MTL regions resulted in a recognition deficit that was most prominent for remember reports, knowing accuracy was apparently unaffected by the damage. However, it should be noted that the data were scored assuming that "remembering" and "knowing" are exclusive processes. Had they been scored assuming redundancy or independence, the tabled data suggest that a deficit might well have been observed for knowing [54]. Similarly, Yonelinas et al. [55] documented small but reliable deficits in familiarity based memory as a consequence of MTL damage when recollection and familiarity are assumed to represent independent processes [55]. In the current study, the selectivity of the success effect to source recognition provides a re- trieval complement to recent evidence that the magnitude of hippocampal activation during relational encoding, but not during item-based learning, correlates with subsequent recognition memory [56].

In contrast to the left prefrontal, parietal, and medial temporal regions differentially associated with source retrieval, we observed right frontopolar and dorsal PFC regions differentially associated with the recency task, and a more lateral right PFC region that demonstrated an increased response on unsuccessful versus successful recency trials. Earlier neuropsychological evidence for the necessity of right PFC in judgments of recency was provided by Milner et al. [57]. In that study, a sub-sample of right frontal patients with damage encompassing all three of the regions noted above was consistently impaired on judgments of recency for concrete words, line drawings, and abstract figures [57]. We suggest that activation in these regions (Fig. 5) correlates with the degree to which subjects must closely monitor modest differences in item familiarity, an orientation that is particularly important when contextual recollection is unavailable or non-diagnostic. This interpretation is consistent with (a) demonstrations that involvement of right frontopolar and dorsolateral PFC during yes-no recognition appears to depend on the subject's expectancy regarding the ratio of old to new items on the test list [24], (b) neuropsychological evidence showing an inordinately high false recognition rate in a patient with right PFC damage [58,59], (c) ERP and fMRI evidence implicating right PFC with acontextual "know" and low confidence recognition responses $[21,25,60]$, and (d) ERP data showing preferential involvement at electrodes over right PFC during discrimination following shallow compared to deep encoding [13].

Although the present behavioral and fMRI data are consistent with our hypothesis that the recency task depends on differential item strength or familiarity, we cannot rule out the possibility that these recency judgments instead depend on some type of temporal recollection. If so, this recollective process must somehow recover list-wide temporal information, and in contrast to source recollection, must be less impaired by speeding retrieval while simultaneously being more impaired by reducing study lag. At present, it is difficult to specify the precise declarative content of this temporal recollective knowledge. Regardless, this characterization of the recency judgment would still require the interpretation that there are systematic retrieval orientation differences between the source and recency tasks, and that these differences depend on separable neural computations that may be preferentially carried out in right PFC for judgments of recency versus left PFC for judgments of source. Elucidation of the exact nature of the information used during the current recency task will require further research and may benefit from comparison with other judgments predicted under dual process theory to heavily rely upon familiarity, such as judgments of prior item frequency.

The present data are consistent with the hypothesis that PFC and parietal regions are critical in mediating the 
manner in which test items are employed as cues for memory. That is, these regions appear to be important for the flexible adoption of decision and monitoring processes that qualitatively differ depending on situational factors [24] such as whether or not item specific recollection is essential for a given task [61]. Such factors have often been overlooked or not fully considered in many prior imaging reports that have contrasted functional data across different stimulus histories (e.g. old versus new items), or different stimulus conditions (e.g. high versus low density lure conditions). The current data indicate that subjects clearly may engage memory differently under such circumstances and therefore it is unclear whether many previously reported differences, especially in PFC, are attributable to differences in the adopted retrieval orientation, the successful recovery of information, or the post-retrieval evaluation of information [62]. Gaining additional leverage on these factors will be imperative in future investigations aimed at further elucidating the mechanisms underlying episodic recognition capabilities and limitations.

\section{Acknowledgements}

Supported by grants from the NIH (P50-NS26985, RO1-MH60941, R03-DC04466, AG08441, and DC04466), the Ellison Medical Foundation, and the McKnight Endowment Fund for Neuroscience. We thank Steve Prince, Matt Hutson and Heather Foley for assistance with data collection, and Russ Poldrack for insightful discussion.

\section{References}

[1] Atkinson RC, Juola JG. Search and decision rpocesses in recognition memory. In: Atkinson RC, Luce RD, Krantz DH, Suppes P, editors. Contemporary developments in mathematical psychology. I. Learning, memory, and thinking. San Francisco: Freeman; 1974. p. 243-93.

[2] Banks WP. Recognition and source memory as multivariate decision processes. Psychological Science 2000;11(4):267-73.

[3] Dobbins IG, Khoe W, Yonelinas AP, Kroll NEA. Predicting individual false alarm rates and signal detection theory: a role for remembering. Memory \& Cognition 2000;28(8):1347-56.

[4] Gardiner JM, Java RI. Recognising and remembering. In: Collins AF, Gathercole SE. et al., editors. Theories of memory. Hillsdale (NJ, US): Lawrence Erlbaum Associates Inc.; 1993. p. 163-88.

[5] Jacoby LL. A process dissociation framework: separating automatic from intentional uses of memory. Journal of Memory \& Language 1991;30(5):513-41.

[6] Mandler G. Recognizing: the judgment of previous occurrence. Psychological Review 1980;87(3):252-71.

[7] Nobel PA, Shiffrin RM. Retrieval processes in recognition and cued recall. Journal of Experimental Psychology: Learning, Memory, \& Cognition 2001;27(2):384-413.

[8] Tulving E. Memory and consciousness. Canadian Psychology 1985;26(1):1-12.

[9] Wagner AD, Gabrieli JDE, Verfaellie M. Dissociations between familiarity processes in explicit recognition and implicit perceptual memory. Journal of Experimental Psychology: Learning, Memory, \& Cognition 1997;23(2):305-23.
[10] Yonelinas AP. Receiver-operating characteristics in recognition memory: evidence for a dual-process model. Journal of Experimental Psychology: Learning, Memory, \& Cognition 1994;20(6):1341-54.

[11] Cabeza R, Mangels J, Nyberg L, Habib R, Houle S, McIntosh AR, et al. Brain regions differentially involved in remembering what and when: a PET study. Neuron 1997;19(4):863-70.

[12] Zorrilla LT, Aguirre GK, Zarahn E, Cannon TD, D'Esposito M. Activation of the prefrontal cortex during judgments of recency: a functional MRI study. Neuroreport 1996;7(15-17):2803-6.

[13] Rugg MD, Allan K, Birch CS. Electrophysiological evidence for the modulation of retrieval orientation by depth of study processing. Journal of Cognitive Neuroscience 2000;12(4):664-78.

[14] Henson RN, Shallice T, Dolan RJ. Right prefrontal cortex and episodic memory retrieval: a functional MRI test of the monitoring hypothesis. Brain 1999;122(Pt 7):1367-81.

[15] Nolde SF, Johnson MK, D'Esposito M. Left prefrontal activation during episodic remembering: An event-related fMRI study. Neuroreport: An International Journal for the Rapid Communication of Research in Neuroscience 1998;9(15):3509-14.

[16] Rugg MD, Fletcher PC, Allan K, Frith CD, Frackowiak RS, Dolan RJ. Neural correlates of memory retrieval during recognition memory and cued recall. Neuroimage 1998;8(3):262-73.

[17] Ranganath C, Johnson MK, D'Esposito M. Left anterior prefrontal activation increases with demands to recall specific perceptual information. Journal of Neuroscience 2000;20(22):RC108.

[18] Donaldson DI, Petersen SE, Buckner RL. Dissociating memory retrieval processes using fMRI: Evidence that priming does not support recognition memory. Neuron 2001;31:1047-59.

[19] Konishi S, Wheeler ME, Donaldson DI, Buckner RL. Neural correlates of episodic retrieval success. Neuroimage 2000;12(3):27686.

[20] McDermott KB, Jones TC, Petersen SE, Lageman SK, Roediger III HL. Retrieval success is accompanied by enhanced activation in anterior prefrontal cortex during recognition memory: an event-related fMRI study. Journal of Cognitive Neuroscience 2000;12(6):965-76.

[21] Henson RN, Rugg MD, Shallice T, Josephs O, Dolan RJ. Recollection and familiarity in recognition memory: an event-related functional magnetic resonance imaging study. Journal of Neuroscience 1999;19(10):3962-72.

[22] Lepage M, Ghaffar O, Nyberg L, Tulving E. Prefrontal cortex and episodic memory retrieval mode. Proceedings of the National Academy of Sciences of the United States of America 2000;97(1):506-11.

[23] Schacter DL, Buckner RL, Koutstaal W, Dale AM, Rosen BR. Late onset of anterior prefrontal activity during true and false recognition: an event-related fMRI study. Neuroimage 1997;6(4):259-69.

[24] Wagner AD, Desmond JE, Glover GH, Gabrieli JD. Prefrontal cortex and recognition memory: functional-MRI evidence for context-dependent retrieval processes. Brain 1998;121(10):19852002.

[25] Henson RN, Rugg MD, Shallice T, Dolan RJ. Confidence in recognition memory for words: dissociating right prefrontal roles in episodic retrieval. Journal of Cognitive Neuroscience 2000;12(6):913-23.

[26] Aggleton JP, Brown MW. Episodic memory, amnesia, and the hippocampal-anterior thalamic axis. Behavioral \& Brain Sciences 1999;22(3):425-89.

[27] Cabeza R, Rao SM, Wagner AD, Mayer AR, Schacter DL. Can medial temporal lobe regions distinguish true from false? An event-related functional MRI study of veridical and illusory recognition memory. Proceedings of the National Academy of Sciences of the United States of America 2001;98(8):4805-10.

[28] Eldridge LL, Knowlton BJ, Furmanski CS, Bookheimer SY, Engel SA. Remembering episodes: a selective role for the hippocampus during retrieval. Nature Neuroscience 2000;3(11):1149-52. 
[29] McClelland JL, McNaughton BL, O'Reilly RC. Why there are complementary learning systems in the hippocampus and neocortex: insights from the successes and failures of connectionist models of learning and memory. Psychological Review 1995;102(3):419-37.

[30] Nyberg L, McIntosh AR, Houle S, Nilsson LG, Tulving E. Activation of medial temporal structures during episodic memory retrieval (see comments). Nature 1996;380(6576):715-7.

[31] Schacter DL, Alpert NM, Savage CR, Rauch SL, Albert MS Conscious recollection and the human hippocampal formation: evidence from positron emission tomography. Proceedings of the National Academy of Sciences of the United States of America 1996;93(1):321-5.

[32] Squire LR. Memory and the hippocampus: a synthesis from findings with rats, monkeys, and humans. Psychological Review 1992;99(2):195-231.

[33] Moscovitch M. Memory and working-with-memory: a component process model based on modules and central systems. Journal of Cognitive Neuroscience 1992;4(3):257-67.

[34] Moscovitch M, Umilta C. Conscious and nonconscious aspects of memory: a neuropsychological framework of modules and central systems. In: Lister RG, Weingartner HJ, editors. Perspectives on cognitive neuroscience. New York (NY, US): Oxford University Press; 1991. p. 229-66

[35] Brown MW, Aggleton JP. Recognition memory: what are the roles of the perirhinal cortex and hippocampus? Natural Review of Neuroscience 2001;2(1):51-61.

[36] Mandler G, Boeck WJ. Retrieval processes in recognition. Memory \& Cognition 1974;2(4):613-5.

[37] McElree B, Dolan PO, Jacoby LL. Isolating the contributions of familiarity and source information to item recognition: a time course analysis. Journal of Experimental Psychology: Learning, Memory, \& Cognition 1999;25(3):563-82.

[38] Kucera H, Francis WN. Computational analysis of present-day American English. Providence: Brown University Press; 1967.

[39] Murdock BB, Dufty PO. Strength theory and recognition memory. Journal of Experimental Psychology 1972;94(3):284-90.

[40] Hockley WE. Recognition memory for item and associative information: a comparison of forgetting rates. In: Hockley WE, Lewandowsky S, editors. Relating theory and data: essays on human memory in honor of Bennet B. Murdock. Hillsdale (NJ, US) Lawrence Erlbaum Associates, Inc.; 1991. p. 227-48.

[41] Hintzman DL, Curran T. Retrieval dynamics of recognition and frequency judgments: evidence for separate processes of familiarity and recall. Journal of Memory \& Language 1994;33(1):1-18.

[42] Cohen JD, MacWhinney B, Flatt M, Provost J. PsyScope: an interactive graphic system for designing and controlling experiments in the psychology laboratory using Macintosh computers. Behavior Research Methods Instruments \& Computers 1993;25(2):257-71.

[43] Dale AM, Greve DN, Burock MA. Optimal stimulus sequences for event-related fMRI. In: Proceedings of the Presentation at the Fifth International Conference on the Functional Mapping of the Human Brain. Germany: Duesseldorf; 1999 June. p. 22-6.

[44] Schacter DL, Wagner AD. Medial temporal lobe activations in fMRI and PET studies of episodic encoding and retrieval. Hippocampus 1999;9(1):7-24.
[45] Strange BA, Fletcher PC, Henson RN, Friston KJ, Dolan RJ. Segregating the functions of human hippocampus. Proceedings of the National Academy of Sciences of the United States of America 1999;96(7):4034-9.

[46] Strange BA, Otten LJ, Josephs O, Rugg MD, Dolan RJ. Dissociable human perirhinal, hippocampal and parahippocampal roles during verbal encoding. Journal of Neuroscience 2002;22(2):523-8.

[47] Rugg MD, Fletcher PC, Chua PM, Dolan RJ. The role of the prefrontal cortex in recognition memory and memory for source: an fMRI study. Neuroimage 1999;10(5):520-9.

[48] Buckner RL, Koutstaal W, Schacter DL, Wagner AD, Rosen BR. Functional-anatomic study of episodic retrieval using fMRI. I. Retrieval effort versus retrieval success. Neuroimage 1998;7(3):15162

[49] Maril A, Wagner A, Schacter D. On the tip of the tongue: an event-related fMRI study of semantic retrieval failure and cognitive conflict. Neuron 2001;31:653-60.

[50] Raye CL, Johnson MK, Mitchell KJ, Nolde SF, et al. fMRI investigations of left and right PFC contributions to episodic remembering. Psychobiology 2000;28(2):197-206.

[51] Dobbins IG, Foley H, Schacter DL, Wagner AD. Executive control during retrieval: multiple prefrontal processes subserve source memory. Neuron 2002;35(5):989-96.

[52] Dobbins IG, Kroll NEA, Yonelinas AP, Liu Q. Distinctiveness in recognition and free recall: the role of recollection in the rejection of the familiar. Journal of Memory \& Language 1998;38(4):381-400.

[53] Moscovitch DA, McAndrews MP. Material-specific deficits in "remembering" in patients with unilateral temporal lobe epilepsy and excisions. Neuropsychologia 2002;40(8):1335-42.

[54] Yonelinas AP, Jacoby LL. The relation between remembering and knowing as bases for recognition: effects of size congruency. Journal of Memory \& Language 1995;34(5):622-43.

[55] Yonelinas AP, Kroll NEA, Dobbins I, Lazzara M, Knight RT. Recollection and familiarity deficits in amnesia: convergence of remember-know, process dissociation, and receiver operating characteristic data. Neuropsychology 1998;12(3):323-39.

[56] Davachi L, Wagner AD. Hippocampal contributions to episodic encoding: insights from relational and item-based learning. Journal of Neurophsyiology 2002;88(2):982-90.

[57] Milner B, Corsi P, Leonard G. Frontal-lobe contribution to recency judgements. Neuropsychologia 1991;29(6):601-18.

[58] Curran T, Schacter DL, Norman KA, Galluccio L. False recognition after a right frontal lobe infarction: memory for general and specific information. Neuropsychologia 1997;35(7):1035-49.

[59] Schacter DL, Curran T, Galluccio L, Milberg WP, Bates JF. False recognition and the right frontal lobe: a case study. Neuropsychologia 1996;34(8):793-808.

[60] Duzel E, Yonelinas AP, Mangun GR, Heinze HJ, Tulving E. Event-related brain potential correlates of two states of conscious awareness in memory. Proceedings of the National Academy of Sciences of the United States of America 1997:94(11):5973-8.

[61] Johnson MK, Hashtroudi S, Lindsay DS. Source monitoring. Psychological Bulletin 1993;114(1):3-28.

[62] Rugg MD, Wilding EL. Retrieval processing and episodic memory. Trends in Cognitive Sciences 2000;4(3):108-15. 